\title{
BAKKER CLASSIFICATION IN TREATMENT OF SACRAL STRESS FRACTURES: A SINGLE CENTER EXPERIENCE
}

\author{
(1) Sinan BAHADIR ${ }^{1}$, (1) Vugar NABi², (1) Selim AYHAN³, (1) Selçuk PALAOĞLU4 ${ }^{4}$, (1) Emre ACAROĞLU5
}

\author{
${ }^{1}$ Amasya University, Department of Neurosurgery, Amasya, Turkey \\ ${ }^{2}$ University of Health Sciences Turkey, Antalya Training and Research Hospital, Department of Orthopaedics and Traumatology, Antalya, Turkey \\ ${ }^{3}$ Başkent University Hospital, Department of Neurosurgery, Ankara, Turkey \\ ${ }^{4}$ ARTES Spine Center at Acıbadem Ankara Hospital, Ankara, Turkey \\ ${ }^{5}$ Ankara Spine Center, Ankara, Turkey
}

\begin{abstract}
Objective: Sacral stress fractures mostly occur in patients with a compromised bone structure. Both surgical and nonsurgical modalities are used in the treatment of these patients. However, there is not a well-established treatment guideline.

The purpose of this study was to evaluate whether the Bakker classification can be used as a guide when selecting a treatment modality for the treat sacral stress fractures.

Materials and Methods: This retrospective study assessed 19 consecutive patients who were diagnosed with a sacral stress fracture. The patients' demographics, imaging studies, treatment modalities and outcomes were extracted from their records. Imaging studies were re-evaluated according to the Bakker classification, and fractures were classified accordingly. Finally, the distribution of treatment modalities regarding fracture types was evaluated.

Results: Three patients had type A sacral stress fractures, 11 had type B sacral stress fractures. Five patients, four of whom had prior lumbosacral fusion surgery, had type C fractures. All type A cases were relieved by conservative management. Eight type B fractures were treated by percutaneous procedures. All patients with type $\mathrm{C}$ fractures have undergone lumbopelvic fusion. The mean follow-up period was $31.2 \pm 18.9$ months, and a marked reduction in pain was found after all therapeutic approaches.

Conclusion: The management of sacral stress fractures primarily depends on the type of fracture. The authors recommend lumbopelvic fixation for fractures with prior lumbosacral instrumentation and conservative treatment for type A fractures. Though percutaneous sacroplasty is effective in type B fractures, some can be managed by conservative treatment, whereas others require percutaneous sacroiliac fusion. Further prospective studies with larger populations are needed to confirm the suggested fracture classification-based treatment algorithm.

Keywords: Sacrum, stress fracture, insufficiency fracture, treatment, Bakker classification
\end{abstract}

\section{INTRODUCTION}

As life expectancy has increased over the last decades, so has the prevalence of sacral stress fractures (SSF) $)^{(1)}$. SSF is mostly observed in people with underlying diseases that affect the bone structure, especially in osteoporotic individuals ${ }^{(2)}$. It is also reported in patients who underwent spinal fusion ${ }^{(3)}$. Apart from bone structure, SSF may also be related to biomechanical factors, such as hyperlordosis, impaired pelvic ring stability, repetitive microtrauma and weight gain(4,5).

SSF is both a diagnostic and a treatment challenge for the physician. Symptoms are usually non-specific and vary from low back pain and tenderness to sacral radiculopathy. A prolonged lower back pain - with or without radiation to the buttocks, hip or groin - in physically active patients without trauma history or immobile patients with a low energy trauma history requires an investigation regarding $\mathrm{SSF}^{(6)}$.

The complex pathogenesis involving both bone structure and biomechanical factors make the treatment of SSF challenging. Also, the expectation of patients, patient's prior lifestyle, comorbidities, duration of pain and fracture morphology should be considered in the treatment decision. Conservative management, including immobilisation and analgesics, is generally the first choice of treatment modality ${ }^{(7)}$. For those patients in whom conservative management failed, percutaneous sacroplasty, iliosacral fixation and spinopelvic fixation are some of the available surgical modalities ${ }^{(8-10)}$. Given the high complication rates, mobilisation of patients as early as possible is advocated regardless of treatment modality ${ }^{(11)}$. However, there is no widely accepted treatment algorithm. 
Treatment is mostly up to the treating physician's preference. To date, SSFs have been classified either in conjunction with pelvic ring fractures or separately by some authors ${ }^{(12-16)}$. However, they were based on a younger population, were not specific to SSFs and did not define fracture morphology relative to the sacral foramina, which is an important anatomical structure regarding surgical procedures. Recently, Bakker et al. ${ }^{(17)}$ proposed a new classification for SSFs since they found that the existing classification systems were inadequate for use in decision making for sacroplasty.

The aim of this retrospective study was to re-evaluate our patients' data, classify the fractures according to the Bakker classification and assess treatments and their outcomes regarding fracture types.

\section{MATERIALS AND METHODS}

Data of nineteen consecutive patients who were diagnosed with SSF and treated accordingly in our institute between April 2013 and March 2018 were analysed retrospectively. All patients provided written consent to be enrolled in the study. The treatment modality was decided by the treating physician. The mean follow-up period was $31.2 \pm 18.9$ months. The following data were collected from electronic patient records and the hospital-based data archiving and communication system: patients' demographics, imaging studies, treatment modality (conservative vs surgical), type of surgical procedure if surgery was performed and outcome scores. A visual analogue scale (VAS) is in routine use at our centre, and patients are assessed at each visit. The scores on the VAS are used as outcome scores. VAS scores of the study population were assessed for low back pain before the treatment and at follow-up visits. Magnetic resonance imaging (MRI) of patients were re-evaluated, and fractures were classified according to Bakker et al.'s ${ }^{(17)}$ study (Table 1).

\section{RESULTS}

In the study population, the mean age was $50.9 \pm 16.7$ years, and the mean body mass index was $26.2 \pm 1.3 \mathrm{~kg} / \mathrm{m}^{2}$. Of 19 patients,
11 were female. All patients had acute onset low back pain that radiated to the posterior thigh and worsened while standing or walking. All patients described a new onset of difficulty walking. The mean VAS score of the patient population was $76.5 \pm 11$ before the treatment, $57 \pm 9,46.5 \pm 9.6$ and $27.5 \pm 6.9$ in the sixth week, third month and sixth month, respectively after the beginning of treatment. This regression in VAS score was statistically significant at all intervals $(p=0.001)$.

When fractures were classified, three of 19 patients had type A fractures, and all were relieved with conservative treatment (Figure 1a, b). Eleven patients had type B fractures (Figure 1c, d). Six of these patients required percutaneous sacroplasty, whereas three patients were managed successfully with conservative treatment. The remaining two patients required percutaneous sacroiliac fixation (Figure 1e, f). Five patients had type $C$ fractures. Four have undergone lumbosacral instrumentation previously. All five patients were treated with lumbopelvic fusion (Figure 1g, h) The distribution of patients and treatment modalities regarding the type of fractures are summarised in Table 2.

\section{DISCUSSION}

SSF, first described by Lourie, are an important cause of low back pain ${ }^{(18)}$. They can be either "fatigue" fractures resulting from abnormal repeated stress on healthy bones or "insufficiency" fractures due to the inability of a bone with deficient elastic resistance to withstand physiologic stress ${ }^{(17,19)}$. Insufficiency fractures are mostly associated with osteopenia/osteoporosis in the elderly. Finally, it is known that short lumbosacral fusion or long-segment fusion may precede SSF, especially in older females with instrumentation ${ }^{(20,21)}$. In the present study, the patients lacked a bone mineral density test, so we were unable to subclassify the fractures. However, most of the population were older than 65 years old, and no patient had a high-energy trauma history. Thus, we think that most, if not all, fractures in the dataset can be classified as insufficiency fractures.

The presence of prolonged lower back pain, with or without radiation to the buttocks, hip or grain, or local tenderness with

\section{Table 1. Summary of Bakker's Classification}

\begin{tabular}{|c|c|c|c|}
\hline Type & Subtype & Region & Characteristics \\
\hline \multirow{3}{*}{ A } & A1 & \multirow{3}{*}{ Ala } & Bone bruise in MRI with no cortical disruption in CT scan \\
\hline & A2 & & Deformation of the anterior cortical bone without disruption of the cortex \\
\hline & A3 & & Compression fractures of the anterolateral rim of ala \\
\hline \multirow{3}{*}{ B } & B1 & \multirow{3}{*}{ Ala } & Fracture is parallel to the sacroiliac joint \\
\hline & B2 & & Fracture involves the sacroiliac joint \\
\hline & B3 & & Fracture line involves the neural foramina \\
\hline \multirow{3}{*}{ C } & $\mathrm{C} 1$ & \multirow[b]{3}{*}{ Corpus } & Fractures extend from the anterior cortical bone to the dorsal corpus \\
\hline & C2 & & Fractures extend into the neural foramina or the spinal canal unilaterally \\
\hline & C3 & & Horizontal fractures of the corpus with bilateral sagittal completion \\
\hline
\end{tabular}


no obvious pathology should direct the physician to a possible SSF. The MRI is the most sensitive imaging modality for the diagnosis of stress fractures ${ }^{(22)}$. However, computed tomography (CT) can demonstrate fracture morphology in more detail in the presence of cortical disruption ${ }^{(19)}$.

For treatment, there is not a universally accepted guideline, and treatment is mostly decided on a case by case basis ${ }^{(12,13,16)}$. Since most SSF cases are stable fractures, conservative management has been the primary treatment modality. Bedrest, analgesics and physical therapy alone or in combination, was shown to have good functional outcomes ${ }^{(23)}$.

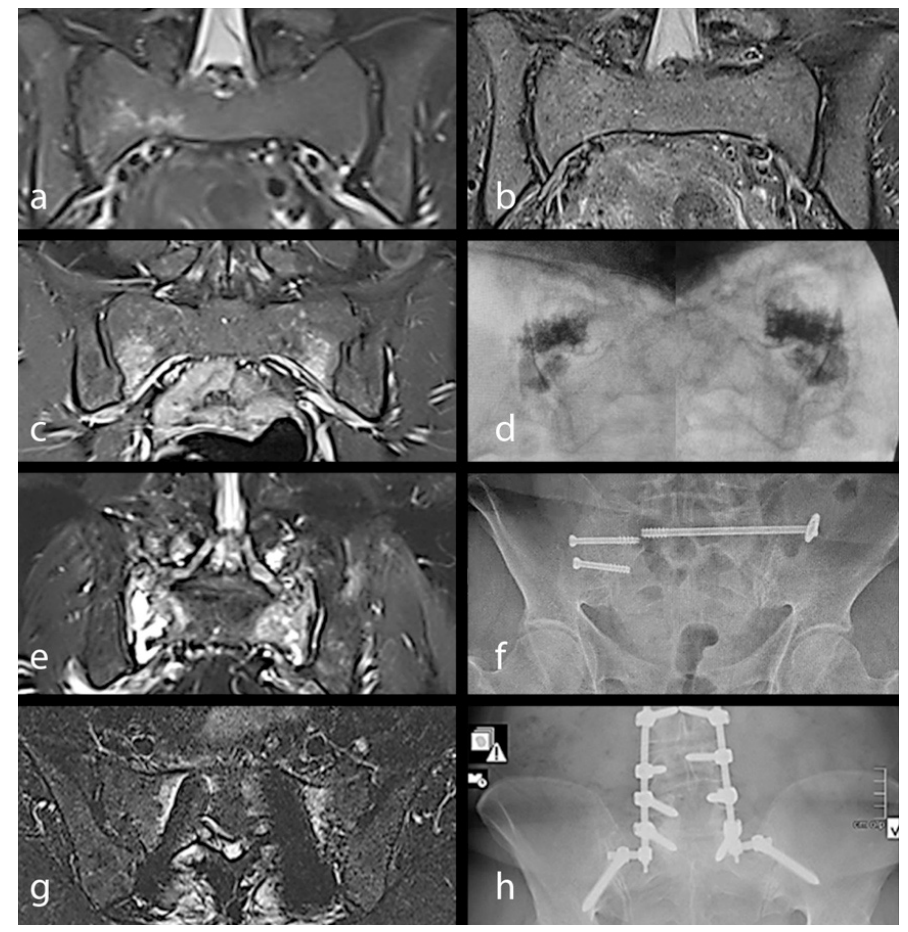

Figure 1. Coronal T2-weighted (T2W) short tau inversion recovery (STIR) sequence demonstrating hyperintensity located within the right sacral ala (a). The fracture is healed with conservative treatment (b). Hyperintensity in coronal T2W STIR sequence suggestive of bilateral sacral alar fracture (c). Percutaneous sacroplasty achieves functional improvement (d). Bilateral alar fractures are seen as hyperintensity zones on MRI (e) are treated successfully by bilateral percutaneous sacroiliac fixation (f). Sacral stress fracture accompanying lumbosacral instrumentation (g) can be treated with lumbopelvic fusion (h)
When conservative management fails or the immediate control of pain and rapid mobilisation is the goal, surgical procedures are considered. With sacroplasty, a significant reduction in pain and improvement in mobility can be achieved in patients with an SSF $^{(24,25)}$. Another treatment method for nondisplaced SSF is the stabilisation with minimally invasive sacroiliac (SI) screws that are inserted percutaneously through the SI joint and fixed to the vertebral body of S1 or S2(26). In all SSF cases following lumbar spinal fusion, lumbopelvic instrumentation is advised to avoid risk factors and activity modification ${ }^{(9)}$.

Recently, Bakker et al.(17) proposed a classification system for sacral insufficiency fractures based on CT findings since previous classification systems do not provide sufficient insight regarding the relation of fracture lines to neural foramina, which is important during sacroplasty. The classification is summarised in Table 1. They considered sacroplasty as feasible in SSFs of types A and B. On the other hand, the C3 subgroup is unstable and may require open reduction and stabilisation. They consider the Bakker classification to be useful in differentiating fractures of less mechanical importance and assessing risk for cement leakage toward neural structures during sacroplasty.

The patients in this retrospective study were diagnosed by lumbar and pelvic MRI. Treatment did not follow a specific algorithm but was decided on a case by case basis. However, as a general approach, treatments progressed from the most conservative to the most invasive as they failed. In cases of unstable SSFs or those following lumbar spinal instrumentation, a lumbopelvic stabilisation was performed without any prior treatment. In our daily practice, regardless of treatment modality, all treated patients were mobilised rapidly without any activity modification following their respective treatments. When the fractures were re-evaluated based on Bakker's classification, we found that all patients with type A fracture were successfully treated with conservative methods. All patients showed improved VAS scores and functional improvement in the short term when compared with their pre-treatment evaluation. However, our sample size was small, and there might be patients with type A fractures who will be refractory to conservative treatments.

The majority of patients in our series $(n=11)$ had type $B$ fractures. Most of them were treated effectively with

Table 2. Satisfactory treatment modalities regarding fracture types

\begin{tabular}{|c|c|c|c|c|}
\hline & & Fractur & & \\
\hline & & A & B & C \\
\hline Patients & Overall (F:M) & $3(2: 1)$ & $11(5: 6)$ & $5(4: 1)$ \\
\hline & Conservative & 3 & 3 & - \\
\hline & Percutaneous sacroplasty & - & 6 & - \\
\hline
\end{tabular}


percutaneous sacroplasty. All but three patients were relieved with conservative treatment, and two required percutaneous stabilisation of the sacroiliac joint. Further studies with larger sample sizes might reveal factors (i.e. subtype of the fracture, age, bone mineral density, and others) that are associated with satisfactory results with conservative management in this type of fracture.

In our series, five patients had type $C$ fractures. Four patients have undergone lumbosacral instrumentation previously. All but one patient has received treatments previously without satisfactory outcomes. All patients showed functional improvement and a decrease in pain scores with sacroiliac fixation. Since there was one isolated type $\mathrm{C}$ fracture, we cannot generalise this treatment for type $\mathrm{C}$ fractures.

\section{Study Limitations}

This study is not devoid of limitations. First, this is a retrospective study, and though all consecutive patients with SSF were included, no strict diagnostic and treatment protocols were used in decision making. Second, though the authors used the insufficiency fracture classification proposed by Bakker et al. ${ }^{(17)}$, there is no supporting evidence that cases in this study were insufficiency fractures. Another limitation is the small population size but considering the incidence and difficulty in the diagnosis of SSF, any report on the subject would be helpful for further studies.

\section{CONCLUSION}

Conservative management is recommended as the first line of treatment in type A fractures. Percutaneous sacroplasty and sacroiliac fixation are effective treatment methods for type $B$ fractures. However, physicians should also consider conservative management since not all of them require invasive procedures. Our data is not sufficient to make any recommendation for type C fractures. We recommend sacroiliac fixation for SSFs with previous lumbosacral fusion surgery since most patients show no improvement with other treatments.

\section{Ethics}

Ethics Committee Approval: Retrospective study. Informed Consent: Retrospective study.

\section{Authorship Contributions}

Concept: S.B., V.N., S.A., S.P., E.A., Design: S.B., V.N., S.A., S.P., E.A., Data Collection or Processing: V.N., S.A., Analysis or Interpretation: S.B., V.N., S.A., E.A., Literature Search: S.B., V.N., S.A., Writing: S.B., V.N., S.A.

Conflict of Interest: No conflict of interest was declared by the authors.

Financial Disclosure: The authors declared that this study received no financial support.

\section{REFERENCES}

1. Kanis JA, Johnell O, Oden A, Jonsson B, De Laet C, Dawson A. Risk of hip fracture according to the World Health Organization criteria for osteopenia and osteoporosis. Bone. 2000;27:585-90.

2. Warriner AH, Patkar NM, Curtis JR, Delzell E, Gary L, Kilgore M, et al. Which fractures are most attributable to osteoporosis? Journal of clinical epidemiology. 2011;64:46-53.

3. Meredith DS, Taher F, Cammisa FP, Jr., Girardi FP. Incidence, diagnosis, and management of sacral fractures following multilevel spinal arthrodesis. The spine journal : official journal of the North American Spine Society. 2013;13:1464-9.

4. Richards AM, Coleman NW, Knight TA, Belkoff SM, Mears SC. Bone density and cortical thickness in normal, osteopenic, and osteoporotic sacra. Journal of osteoporosis. 2010;2010.

5. Tamaki Y, Nagamachi A, Inoue K, Takeuchi M, Sugiura K, Omichi Y, et al. Incidence and clinical features of sacral insufficiency fracture in the emergency department. The American Journal of Emergency Medicine. 2017;35:1314-6.

6. Lee YJ, Bong HJ, Kim JT, Chung DS. Sacral insufficiency fracture, usually overlooked cause of lumbosacral pain. Journal of Korean Neurosurgical Society. 2008;44:166-9.

7. Tsiridis E, Upadhyay N, Giannoudis PV. Sacral insufficiency fractures: current concepts of management. Osteoporosis international : a journal established as result of cooperation between the European Foundation for Osteoporosis and the National Osteoporosis Foundation of the USA. 2006;17:1716-25.

8. Frey ME, DePalma MJ, Cifu DX, Bhagia SM, Daitch JS. Efficacy and safety of percutaneous sacroplasty for painful osteoporotic sacral insufficiency fractures: a prospective, multicenter trial. Spine. 2007;32:1635-40.

9. Zhang W, Guo X, Zhang J, Hou C, Liu Q, Tang G. [Treatment of sacral fractures with pedical screw systems fixed between lumbar and ilium]. Zhongguo xiu fu chong jian wai ke za zhi = Zhongguo xiufu chongjian waike zazhi $=$ Chinese journal of reparative and reconstructive surgery. 2010;24:521-4.

10. Collinge CA, Crist BD. Combined Percutaneous lliosacral Screw Fixation With Sacroplasty Using Resorbable Calcium Phosphate Cement for Osteoporotic Pelvic Fractures Requiring Surgery. Journal of orthopaedic trauma. 2016;30(6):e217-22.

11. Weber $M$, Hasler $P$, Gerber $H$. Insufficiency fractures of the sacrum. Twenty cases and review of the literature. Spine. 1993;18:2507-12.

12. Denis F, Davis S, Comfort T. Sacral fractures: an important problem. Retrospective analysis of 236 cases. Clinical orthopaedics and related research. 1988;227:67-81.

13. Tile M. Acute Pelvic Fractures: I. Causation and Classification. The Journal of the American Academy of Orthopaedic Surgeons. 1996;4:143-51.

14. Strange-Vognsen $\mathrm{HH}$, Lebech A. An unusual type of fracture in the upper sacrum. Journal of Orthopaedic Trauma. 1991;5:200-3.

15. Isler B. Lumbosacral lesions associated with pelvic ring injuries. Journal of orthopaedic trauma. 1990;4:1-6.

16. Rommens PM, Hofmann A. Comprehensive classification of fragility fractures of the pelvic ring: Recommendations for surgical treatment. Injury. 2013;44:1733-44.

17. Bakker G, Hattingen J, Stuetzer H, Isenberg J. Sacral Insufficiency Fractures : How to Classify? Journal of Korean Neurosurgical Society. 2018;61(2):258-66.

18. Lourie H. Spontaneous osteoporotic fracture of the sacrum. An unrecognized syndrome of the elderly. Jama. 1982;248:715-7.

19. Wagner D, Ossendorf C, Gruszka D, Hofmann A, Rommens PM. Fragility fractures of the sacrum: how to identify and when to treat 
turkishspine

surgically? European journal of trauma and emergency surgery : official publication of the European Trauma Society. 2015;41:349-62.

20. DeWald C], Stanley T. Instrumentation-related complications of multilevel fusions for adult spinal deformity patients over age 65: surgical considerations and treatment options in patients with poor bone quality. Spine. 2006;31(19 Suppl):S144-51.

21. Klineberg E, McHenry T, Bellabarba C, Wagner T, Chapman J. Sacral insufficiency fractures caudal to instrumented posterior lumbosacral arthrodesis. Spine. 2008;33:1806-11.

22. Lyders EM, Whitlow CT, Baker MD, Morris PP. Imaging and treatment of sacral insufficiency fractures. AJNR American journal of neuroradiology. 2010;31:201-10.
23. Newhouse KE, el-Khoury GY, Buckwalter JA. Occult sacral fractures in osteopenic patients. The Journal of bone and joint surgery American volume. 1992;74:1472-7.

24. Waites MD, Mears SC, Richards AM, Mathis JM, Belkoff SM. A biomechanical comparison of lateral and posterior approaches to sacroplasty. Spine. 2008;33:E735-8.

25. Mears SC, Sutter EG, Wall SJ, Rose DM, Belkoff SM. Biomechanical comparison of three methods of sacral fracture fixation in osteoporotic bone. Spine. 2010;35:E392-5.

26. Routt ML, Jr., Kregor PJ, Simonian PT, Mayo KA. Early results of percutaneous iliosacral screws placed with the patient in the supine position. Journal of orthopaedic trauma. 1995;9:207-14. 